\title{
INDUCED DAMPING ON VIBRATING CIRCULAR PLATES SUBMERGED IN STILL FLUID
}

\author{
MANUEL GASCÓN-PÉREZ \\ Escuela Técnica Superior de Ingeniería Aeronáutica y del Espacio, UPM, \\ 28040 Madrid, Spain \\ manuel.gascon@upm.es \\ PABLO GARCÍA-FOGEDA \\ Escuela Técnica Superior de Ingeniería Aeronáutica y del Espacio, UPM, \\ 28040 Madrid, Spain \\ pablo.garciafogeda@upm.es
}

Received

Revised

\begin{abstract}
When a structure vibrates immersed in a fluid it is known that the dynamic properties of the system are modified. The surrounding fluid will, in general, contributes to the inertia, the rigidity and the damping coefficient of the coupled fluid-structure system. For light structures, like spacecraft antennas, even when the fluid is air the contribution to the dynamic properties can be important. For not so light structures the ratio of the equivalent fluid/structure mass and rigidity can be very small and the fluid contribution could be neglected. For the ratio of equivalent fluid/structure damping both terms are of the same order and therefore the fluid contribution must be studied. The working life of the spacecraft structure would be on space and so without any surrounding fluid. The response of a spacecraft structure on its operational life would be attenuated by the structural damping alone but when the structure is dynamically tested on the earth the dynamic modal test is performed with the fluid surrounding it. The results thus are contaminated by the effects of the fluid. If the damping added by the fluid is of the same order as the structural damping the response of the structure in space can be quite different to the response predicted on earth. It is therefore desirable to have a method able to determine the amount of damping induced by the fluid and that should be subtracted of the total damping measured on the modal vibration test. In this work a method for the determination of the effect of the surrounding fluid on the dynamic characteristics of a circular plate has been developed. The plate is assumed to vibrate harmonically with the vacuum modes and the generalized forces matrix due to the fluid is thus computed. For a compressible fluid this matrix is formed by complex numbers indicating that include terms of inertia, rigidity and damping. The matrix due to the fluid loading is determined by a boundary element method (BEM). The BEM used is of circular rings on the plate surface so the number of elements to obtain an accurate result is very low. The natural frequencies of the system are computed by an iteration procedure one by one and also the damping fluid contribution. Comparisons of the present method with various experimental data and other theories show the efficiency and accuracy of the method for any support condition of the plate.
\end{abstract}

Keywords: circular plate; fluid-structure; natural frequencies; fluid influence on damping.

\section{Introduction}

Fluid-structure interaction problem (FSI) is a growing research field because of the many engineering applications, like for example, the stability of aircraft structures, the vibrations induced on a flexible body, the flow of blood through arteries, interior and exterior acoustic problems etc. The works of Hou et al. [2012], He [2015], He et al. [2014] and He et al. [2012] are good examples of review and applications. 
When the domain of the surrounding fluid is infinite the application of the fluid and structural domain of the finite element methods (FEM) has limitations. The boundary element method (BEM), which requires a discretization of only the boundary of the fluid domain is superior to the FEM for unbounded domains. Applications to FSI problems by the FEM/BEM method can be found in many references for example Rodriguez-Tembleque et al. [2015].

The influence of the surrounding fluid on the dynamic characteristics of structures has been well known for many years. However most of these works were more concerned with underwater applications, such as the sonar of a submarine and therefore the surrounding fluid was considered a liquid (negligible compressibility effects). References McLachlan [1932], Peake et al [1954], Amabili et al. [1995] provide good examples or more recent works as Askari et al. [2013] Tariverdilo et al. [2013], Gascón-Pérez et al [2014].

Spacecraft structures will be submitted to dynamic loads with no external fluid surrounding them. Analytical designers are forced to obtain the value by measurements on modal vibration tests since usually damping is difficult to estimate. However, the measured value of damping will be affected by the influence of the surrounding fluid. An important improvement on the behavior of the structure when subjected to dynamic loads could be obtained if only the damping due to the structure could be considered. To do so a method to determine the damping factor due to the fluid is needed. References Atalalla et al. [1966], Berry et al. [1990], Fowler et al. [1987], Iglesia et al.[1996], Lomas et al. [1977] and also the analysis of the vibroacoustic properties of the coupled fluid-plate by Geng and Li [2012] or Zheng and Wei [2013] investigate on this effect for plates.

These structures, mainly rectangular and circular plates, were modeled as "baffled", embedded in an infinitely rigid plane. Dynamic properties of baffled rectangular plates have been studied in great detail obtaining the acoustic pressure distribution employing the Rayleigh integral equation. Only recent research has been focused on unbaffled plates.

In this work a BEM method for the fluid is developed while for the plate eigenvectors based on the vibration of the plate in vacuum are used to solve the FSI problem. This procedure is commonly used for aeroelastic applications, see for example Dowell and Hall [2001], and for acoustical problems, see Seybert et al [1993]. Thus, the integral formulation of the Helmholtz equation for the pressure field, is combined with a normal mode analysis of the structural response. In this paper, the response of an unbaffled circular plate with arbitrary boundary conditions immersed in a fluid is calculated. The pressure jump across the plate, produced by the loads generated by the plate own vibrations, is calculated. This method is based on Kirchhoff's integral formulation of the Helmholtz equation for the pressure field that uses an elemental solution which satisfies the Sommerfield radiation condition (Skudrzyk, [1971]). The integral equation is solved by means of a collocation technique (Finlayson, [1972]) and the finite part of the singular integral is obtained analytically. The generalized forces due to the fluid loading are determined using the vacuum modes of the plate, obtained analytically, as base functions of the structural displacement. An iteration procedure has been developed to calculate the natural frequencies and the damping coefficients of the plate surrounded by a compressible fluid.

\section{Problem Formulation}

The equation for the deformation of the plate submerged in the fluid is from Leissa [1969]:

$$
D \nabla^{4} w(r, \theta, t)+\rho_{p} h \frac{\partial^{2} w}{\partial t^{2}}=\Delta p(r, \theta, t)
$$

With $D=\frac{E \cdot h^{3}}{12\left(1-v^{2}\right)}$ the flexural rigidity, $E$ the elasticity modulus of the material, $h$ the plate thickness, $v$ the Poisson modulus and $\rho_{p}$ the material density of the plate. 
$\Delta p$ is the pressure jump across the plate, $\Delta p=p_{i}-p_{e}$ where $p_{i}$ and $p_{e}$ are the pressure on the lower and upper sides of the plate surface.

This pressure distribution can be obtained by solving the wave equation on the fluid domain for a still fluid

$$
\frac{1}{a_{\infty}^{2}} \frac{\partial^{2} p}{\partial t^{2}}-\Delta p=0
$$

Application of the momentum equation at the surface of the plate yields the boundary condition

$$
\frac{\partial p}{\partial z}=-\rho_{\infty} \frac{\partial^{2} w}{\partial t^{2}} \text { at } z= \pm 0 \quad \text { and } 0<r<a
$$

For a thin plate placed at the $z=0$ plane, and $a$ being the radius of the plate.

At large distances from the plate, the Sommerfield radiation condition has to be satisfied.

If transient motion has faded out, the motion of both the fluid and the structure would be assumed to be harmonic

$$
w(r, \theta, t)=\tilde{w}(r, \theta) \cdot e^{-i \omega t} \text { and } p(r, \theta, z, t)=\tilde{p}(r, \theta, z) \cdot e^{-i \omega t}
$$

The deformation of the plate, when coupled with the fluid, will be expressed as a linear combination of the normal modes of the plate in vacuum

$$
\tilde{w}(r, \theta)=\sum_{m} \sum_{n} W_{m}^{n}(r) \cdot \cos (m \theta) q_{m}^{n}
$$

Where $q_{m}^{n}$ are the weight coefficients that indicate the contribution of the mode $W_{m}^{n}$ to the deformation of the plate.

The functions $W_{m}^{n}(r)$ have the general expression, Leissa [1969]

$$
W_{m}^{n}(r)=\left[A_{m}^{n} J_{m}\left(\beta_{m}^{n} r\right)+B_{m}^{n} I_{m}\left(\beta_{m}^{n} r\right)\right]
$$

Where $J_{m}$ and $I_{m}$ are Bessel and modified Bessel functions of $m^{\text {th }}$ order, $A_{m}^{n}$ and $B_{m}^{n}$ are unknown constants that depend of the mode order $(m, n)$ and of the boundary conditions of the plate edge and $\beta_{m}^{n}$ is a parameter that depends on the dynamic characteristics of the plate and provides the frequencies of the plate in vacuum. The indexes $m, n$ represent the number of nodal diameters and nodal circles respectively.

Once the assumption of harmonic motion is made, equation (2.2) is expressed as

$$
\Delta \tilde{p}+k^{2} \tilde{p}=0
$$

Where $k=\frac{\omega}{a_{\infty}}$ is the wave number.

If at the boundary condition, equation (2.3), equations (2.4) and (2.5) are substituted, it is obtained

$$
\left.\frac{\partial \tilde{p}(r, \theta, z)}{\partial z}\right|_{z=0}=\rho_{\infty} \omega^{2} \tilde{w}(r, \theta)=\rho_{\infty} \omega^{2} \sum_{n} \sum_{m} W_{m}^{n}(r) \cdot \cos (m \theta) q_{m}^{n}
$$

at $z=0$ and $0<r<a$

Then the pressure modulus can also be expanded in harmonic functions of $\theta$

$$
\tilde{p}(r, \theta, z)=\sum_{m} \sum_{n} P_{m}^{n}(r, z) \cdot \cos (m \theta)
$$

and equation (2.8) can now be expressed as 


$$
\left.\frac{\partial P_{m}^{n}}{\partial z}\right|_{z=0}=\rho_{\infty} \omega^{2} W_{m}^{n}(r) q_{m}^{n} \quad 0 \leq r \leq a
$$

By substitution of equation (2.9) at (2.7) the differential equation for the pressure mode $P_{m}^{n}$ is

$$
\frac{\partial^{2} P_{m}^{n}}{\partial z^{2}}+\frac{\partial^{2} P_{m}^{n}}{\partial r^{2}}+\frac{1}{r} \frac{\partial P_{m}^{n}}{\partial r}-\frac{m^{2}}{r^{2}} P_{m}^{n}+k^{2} P_{m}^{n}=0
$$

Applying Green`s identities taking into account the Helmholtz equation for the pressure field and the Green`s function associated to that equation, the following integral equation for the pressure mode $P_{m}^{n}(r, z)$ is obtained (from references Junger [1972] and Skudrzyk [1971]).

$$
P_{m}^{n}(r, z)=-\frac{1}{4 \pi} \int_{0}^{a} \Delta P_{m}^{n}(\sigma) \frac{\partial}{\partial z}\left(\frac{e^{i k \sqrt{(r-\sigma)^{2}+z^{2}}}}{\sqrt{(r-\sigma)^{2}+z^{2}}}\right) 2 \pi \sigma d \sigma
$$

And applying the boundary condition, equation (2.10), it is obtained

Let

$$
\rho_{\infty} \omega^{2} W_{m}^{n}(r) q_{m}^{n}=-\frac{1}{4 \pi} \int_{0}^{a} \Delta P_{m}^{n}(\sigma) \frac{\partial^{2}}{\partial z^{2}}\left(\frac{e^{i k \sqrt{(r-\sigma)^{2}+z^{2}}}}{\sqrt{(r-\sigma)^{2}+z^{2}}}\right)_{z=0} 2 \pi \sigma d \sigma
$$

$$
\mathrm{K}(r-\sigma, z, k)=\left.\frac{\partial^{2}}{\partial z^{2}}\left(\frac{e^{i k \sqrt{(r-\sigma)^{2}+z^{2}}}}{\sqrt{(r-\sigma)^{2}+z^{2}}}\right)\right|_{z=0}
$$

be the Kernel of the integral equation. The solution to equation (2.13) will be obtained approximately by applying a collocation method.

The plate is divided into $\mathrm{N}$ circular rings being $\Delta r_{j}=r_{j+1}-r_{j}$ the thickness of the $j^{\text {th }}$ ring. At each ring a circle at the radii $\bar{r}_{j}=\frac{r_{j+1}+r_{j}}{2}$ is placed where equation (2.13) is fulfilled.

Then this equation can be expressed for the collocation method

$$
\rho_{\infty} \omega^{2} W_{m}^{n}\left(\bar{r}_{j}\right) q_{m}^{n}=-\frac{1}{2} \sum_{i=1}^{N} \Delta P_{m}^{n}\left(\sigma_{i}\right) \int_{r_{i}}^{r_{i+1}} \mathrm{~K}\left(\bar{r}_{j}-\sigma, 0, k\right) \sigma d \sigma
$$

So finally we end up with a linear system of algebraic equations to determine the pressure mode jump at each ring $\Delta P_{m}^{n}\left(\sigma_{i}\right)$.

To improve the stability of the collocation method, the $N$ rings are divided so all of them have the same area. Therefore, the values of $r_{i}$ are obtained in such a manner that the area of each ring be equal to $\frac{\pi a^{2}}{N}$.

When evaluating the integral

$$
\Lambda_{i j}=-\frac{1}{2} \int_{r_{i}}^{r_{i+1}} \mathrm{~K}\left(\bar{r}_{j}-\sigma, 0, k\right) \sigma d \sigma
$$

special care must be taken of the singularity when $\sigma \rightarrow \bar{r}_{j}$. To avoid this singularity, the Kernel function is split into a regular part and a singular part

$$
\mathrm{K}=\mathrm{K}_{r}+\mathrm{K}_{s}
$$

The singular part is integrated analytically and the regular part is integrated numerically.

The singular part is 


$$
\mathrm{K}_{s}=-\frac{1}{R^{3}}-\frac{k^{2}}{2 R}
$$

Where $R=\sqrt{(r-\sigma)^{2}+z^{2}}$ and $\mathrm{K}_{r}=\mathrm{K}-\mathrm{K}_{s}$

Be $\Lambda_{s i j}$ the value of the singular integral

$$
\Lambda_{s i j}=\frac{1}{2} \int_{r_{i}}^{r_{i+1}}\left(\frac{1}{R^{3}}+\frac{k^{2}}{2 R}\right) \sigma d \sigma
$$

Taking Mangler's principal value, from Mangler [1959], is obtained that

$$
\Lambda_{s i j}=-\frac{1}{4}\left[\frac{\bar{r}_{j}}{\left(r_{i+1}-\bar{r}_{j}\right)^{2}}+\frac{\bar{r}_{j}}{\left(\bar{r}_{j}-r_{i}\right)^{2}}\right]+\frac{k^{2}}{4}\left[2 \bar{r}_{j}+\bar{r}_{j} \operatorname{Ln}\left[\left(\frac{r_{i+1}}{\bar{r}_{j}}-1\right)\left(1-\frac{r_{i}}{\bar{r}_{j}}\right)\right]\right]
$$

Thus finally the system of equations (2.15) is expressed as

$$
\rho_{\infty} \omega^{2} W_{m}^{n}\left(\bar{r}_{j}\right) q_{m}^{n}=\sum_{i=1}^{N}\left(\Lambda_{s i j}+\Lambda_{r i j}\right) \Delta P_{m}^{n}\left(r_{i}\right) \quad j=1 \ldots . N
$$

Where $\Lambda_{r i j}$ is the numerical integration of $\mathrm{K}_{r}$.

In matrix form this system of equations (2.21) can be expressed as

$$
\left[\Lambda_{i j}\right]\left\{\Delta P_{m}^{n}\left(r_{i}\right)\right\}=\rho_{\infty} \omega^{2}\left\{W_{m}^{n}\left(\bar{r}_{j}\right)\right\}
$$

So finally the modal pressure jump is given by

$$
\left\{\Delta P_{m}^{n}\left(r_{i}\right)\right\}=\rho_{\infty} \omega^{2}\left[\Lambda_{i j}\right]^{-1}\left\{W_{m}^{n}\left(\bar{r}_{j}\right)\right\}
$$

The next step is to solve equation (2.1). After substitution of equations (2.4), (2.5) and (2.9), this equation is expressed as

$$
D \tilde{\nabla}^{4} W_{m}^{n}(r)-\rho_{p} h \omega^{2} W_{m}^{n}(r)=\Delta P_{m}^{n}(r)
$$

Where $\tilde{\nabla}^{4}$ is a differential operator of the form $\tilde{\nabla}^{4}=\left(\frac{d^{2}}{d r^{2}}+\frac{1}{r} \frac{d}{d r}-\frac{m^{2}}{r^{2}}\right)^{2}$.

By multiplying equation (2.24) by the mode $W_{u}^{v}$ and integration over the surface of the plate, the following system is obtained

$$
\left[[K]-\omega^{2}\left([M]+\left[M_{F}\right]\right)\right]\{q\}=\{0\}
$$

Where the elements of the three matrices are

$$
\begin{gathered}
K_{m u}^{n v}=D\left\lfloor\nabla^{4} W_{m}^{n} \mid[\Delta S]\left\{W_{u}^{v}\right\}\right. \\
M_{m u}^{n v}=\rho_{p} h\left|W_{m}^{n}\right|[\Delta S]\left\{W_{u}^{v}\right\} \\
M_{\text {Fти }}^{n v}=\rho_{\infty}\left\lfloor W_{m}^{n} \mid\left[\left[\Lambda_{i j}\right]^{-1}\right]^{T}[\Delta S]\left\{W_{u}^{v}\right\}\right.
\end{gathered}
$$

Where $[\Delta S]$ is a diagonal matrix containing the surface area of each ring. Since in this method all the rings have the same area, this matrix can be expressed as $[\Delta S]=\frac{\pi a^{2}}{4 N}[I]$.

It should be noted that the matrix $\left[M_{F}\right]$ for compressible cases is a complex matrix (see equation (2.14)) and will depend on the natural frequencies through the wave number $k=\frac{\omega}{a_{\infty}}$. 
Thus, the solution to equation (2.25) for the determination of the natural frequencies $\omega$ becomes a nonlinear eigenvalue problem since the fluid-mass matrix $\left[M_{F}(\omega)\right]$ depends on the frequency.

An iteration procedure needs to be used to obtain the natural frequencies of the system. The iteration scheme is developed as follows:

1. First, the natural frequencies of the system are computed assuming that the surrounding fluid is incompressible. For this case the matrix $\left[M_{F}\right]$ is independent of $\omega$ and the computation of the natural frequencies becomes a standard method for the computation of eigenvalues.

2. From the solution for incompressible case, a set of $N$ frequencies $\omega_{\text {jincomp. }}$ can be obtained for the coupled incompressible fluid-structure system.

3. For each frequency of step 2, a wave number $k_{j}$ is accordingly defined and a mass matrix $\left[M_{F}\left(k_{j}\right)\right]$ can be calculated.

4. For other values of the wave number $k$, the fluid mass matrix can be obtained by linear interpolation between the two computed matrices $\left[M_{F}\left(k_{j}\right)\right]$ and $\left[M_{F}\left(k_{j+1}\right)\right]$.

5. Starting with the lowest wave number available $k_{1}$, the natural frequencies of the system $\left[-\omega^{2}\left([M]+\left[M_{F}\left(k_{1}\right)\right]\right)+[K]\right]\{q\}=\{0\}$ are computed. Let the lowest natural frequency calculated be $\omega_{1}^{i}$. If $\left|\frac{\omega_{1}^{i}}{a_{\infty}}-k_{1}\right|<\varepsilon$, where $\varepsilon$ is a small number, then we proceed to the computation of the next natural frequency of the system. If the above condition is not satisfied then step five is repeated, taking now a new mass matrix for the fluid, close to the wave number $\frac{\omega_{1}^{i}}{a_{\infty}}$ of the set obtained at step four.

In Figure 1, is presented an schematic diagram, showing the iteration procedure for the calculation of the frequencies.

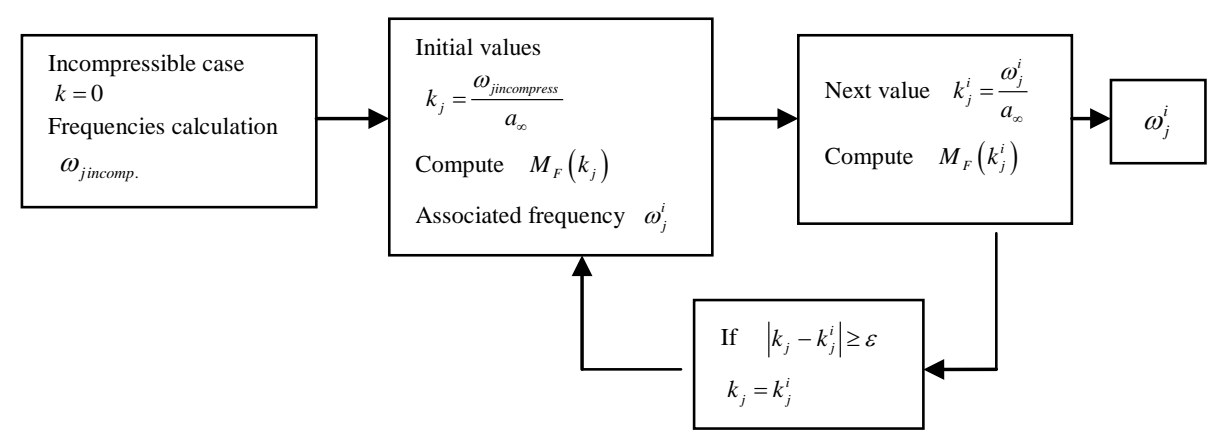

Fig.1. Diagram showing the iteration scheme for the frequencies calculation

For each frequency the above procedure converges in two or three iterations. It should be noted that for a compressible fluid the natural frequencies are obtained one by one, while for an incompressible fluid all of them are obtained at the same time. Once the natural frequencies of the coupled fluid-structure are determined, the normal modes can be computed by determining the 
eigenvector $\left\{q_{i}\right\}$ and expressed as a linear combination of the normal modes of the structure in vacuum.

\section{Results}

For the case of a plate immersed in water (incompressible fluid), in Tables 1, 2 and 3, the frequencies $f_{l m}^{n}$ and $f_{v m}^{n}$ are compared with the results of other authors. The frequency $f_{l m}^{n}$ is associated to the mode $W_{m}^{n}$ of the plate in contact with water, and the frequency $f_{v m}^{n}$ to the corresponding in vacuum, both in $\mathrm{Hz}$.

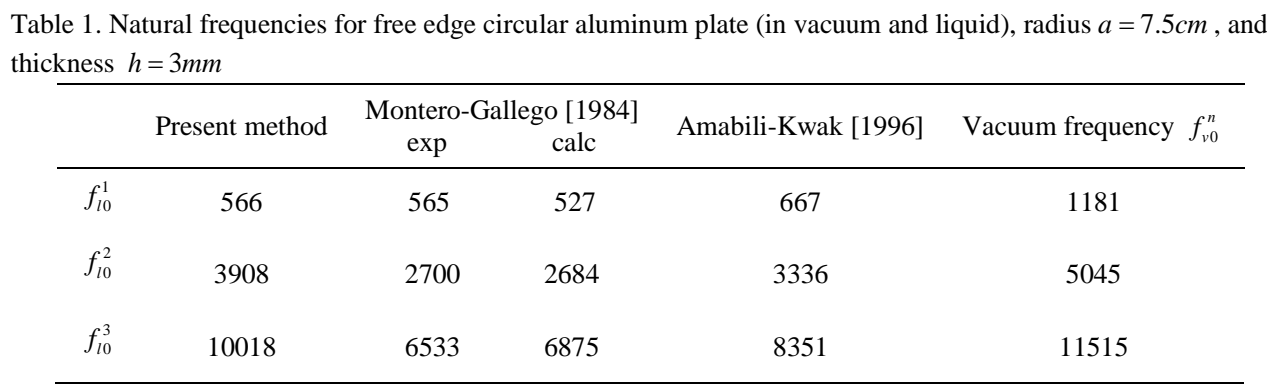

In Table 1, it can be observed that for the first mode, with none nodal circles, the natural frequencies are almost identical between the present method and the experiment of MonteroGallego [1984]. For the second and third mode, the results present differences between the three cases. Probably this can be due to the different boundary condition. In the cases of MonteroGallego [1984] and Amabili-Kwak [1996], the plate is situated in a hole of a rigid wall that separates the fluid on the lower and upper sides of the plate, and even in the case of MonteroGallego [1984] the fluid domain is not infinite because it is immersed in a tank of finite dimensions. In the modes with nodal circles this difference should be more measurable due to the change in sign on the vibration amplitude and therefore on the pressures exerted by the fluid.

In Table 2, the results for the present method are compared with those obtained by Lamb [1920]. Only the fundamental frequency was computed by Lamb, so only this frequency is compared. Both methods provide exactly the same result.

Table 2. Natural frequencies for clamped circular aluminum plate (in vacuum and liquid) radius $a=7.5 \mathrm{~cm}$, thickness $h=3 m m$

\begin{tabular}{ccc}
\hline & Present method & $\begin{array}{c}\text { Lamb } \\
{[1920]}\end{array}$ \\
\hline$f_{10}^{0}$ & 500 & 500 \\
$f_{v 0}^{0}$ & 1327 & 1327 \\
\hline
\end{tabular}

In Table 3 the natural frequencies for a free-edge plate are computed for the cases of zero and one nodal diameter. 


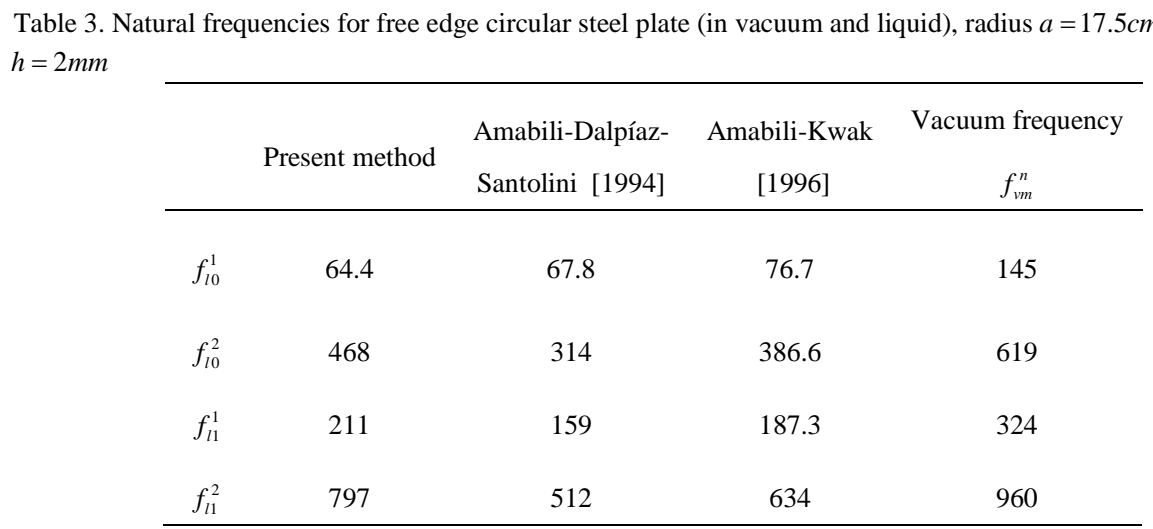

It can be observed that for the first mode the natural frequencies are similar, but for the rest of the modes the results present differences between the three cases with zero and one nodal diameters. This can be due to the different boundary conditions, because in the case of AmabiliKwak [1996], the plate is situated in a hole of a rigid wall that separates the fluid. The rigid wall will make that more kinetic energy of the fluid flows to the vibrating plate thus reducing the natural frequency. In the case of Amabili-Dalpiaz-Santolini [1994] the fluid domain is not infinite because it is immersed in a tank of finite dimension. Further studies should be carried out to see how the reduction of the fluid domain can affect the natural frequencies of the plate.

For the case of the plate immersed in air (compressible fluid), two boundary conditions for the plate are considered: clamped plate and free edge plate. The plate is made of two skins of carbon fiber and a core of honeycomb with the following properties:

Radius $a=1 \mathrm{~m}$. Thickness $h=1 \mathrm{~cm}$. Elasticity modulus $E=9 \times 10^{9} \mathrm{~Pa}$.

Density $\rho_{p}=139 \frac{\mathrm{kg}}{\mathrm{m}^{3}}$. Poisson modulus $v=0.3$

In Figs. 2 and 3, the fluid mass coefficient as function of the wave number $k=\frac{\omega}{a_{\infty}}$ is presented. The coefficient is defined as $C_{m f}=\frac{M_{f}}{4 \pi a^{3} \rho_{\infty}}$ where $M_{f}$ is the diagonal term of the fluid mass matrix that is responsible for the reduction of the frequency in relation to the vacuum value. $C_{m f}$ presents a maximum for intermediate values of the wave number $k$ and for high values of the wave number $k$ tends to reach a zero value, so this fluid mass coefficient can be interpreted as the transfer function that gives the effect of the fluid over the plate (jump of pressure) associated to the deformation mode of the plate. This fluid mass coefficient is constant with the plate radius $a$, because the fluid mass matrix is proportional to the cube of the radius of the plate. 


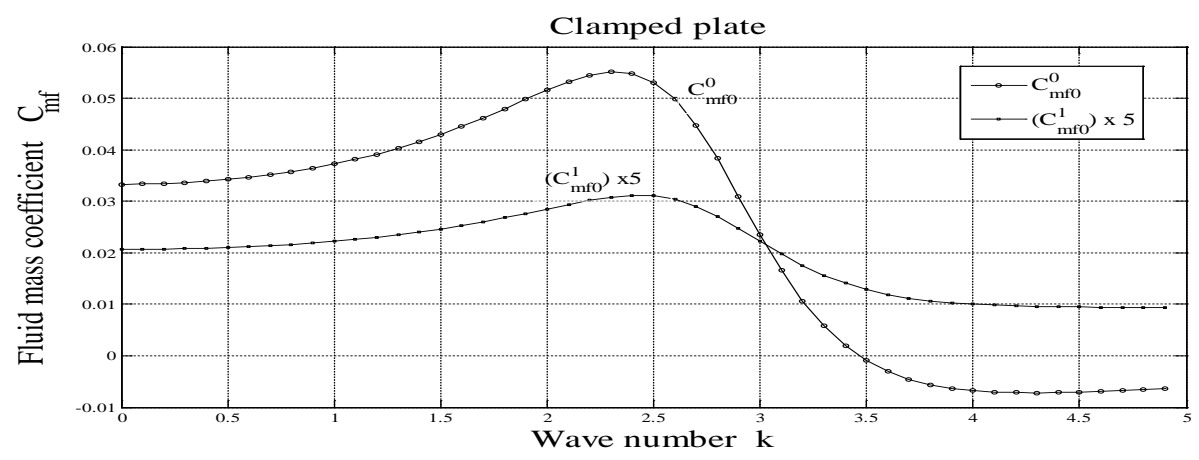

Fig.2. Fluid mass coefficient vs wave number $k$ for a clamped plate

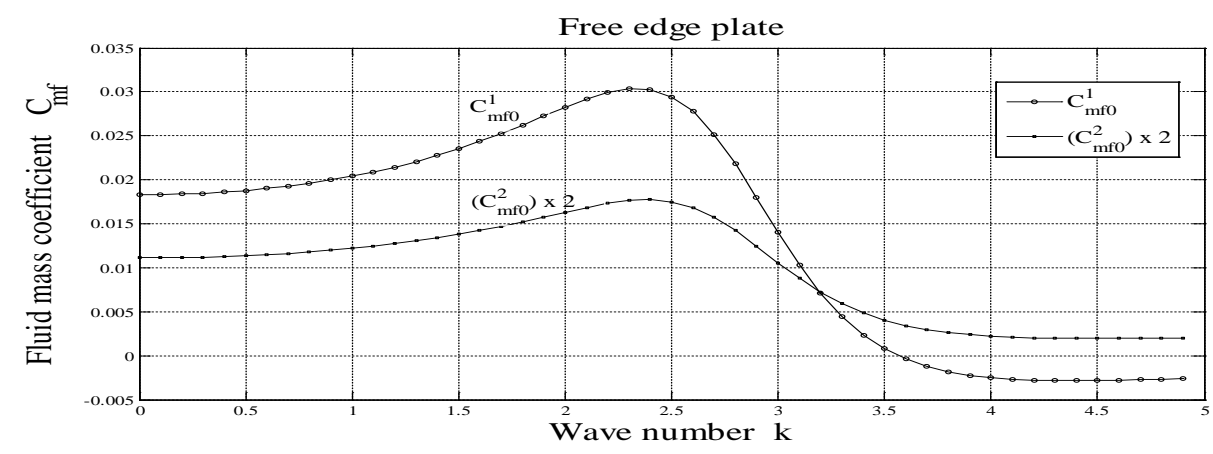

Fig.3. Fluid mass coefficient vs wave number $k$ for a free edge plate

In Figs. 4 and 5, the damping ratio coefficient as function of the wave number $k$ is presented. The coefficient is defined as $\xi=-\frac{\left(M_{f}\right)_{\text {imag }} \cdot \omega}{C_{c r}}$ where $\left(M_{f}\right)_{\text {imag }}$ is the imaginary part of the diagonal term of the fluid mass matrix that is responsible of damping. $C_{c r}$ is the critical damping defined as $C_{c r}=2 \sqrt{K \cdot M_{T}}$ where $K$ and $M_{T}$ are the diagonal terms of the rigidity and total mass matrices. $\xi$ presents a maximum for intermediate values of the wave number $k$ and for high values of $k$, tends to reach a zero value. This damping ratio due to acoustic losses is important for some values of the reduced frequency $k$ and must be taken into account because the value of the structural damping ratio for the material of this sandwich plate can be the order of 0.15 .

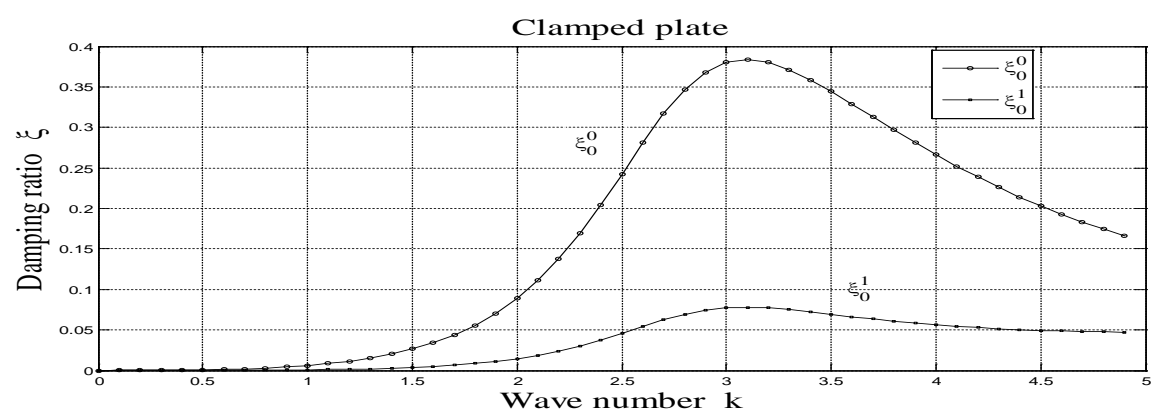

Fig.4. Damping ratio $\xi_{m}^{n}$ vs wave number $k$ for a clamped plate 


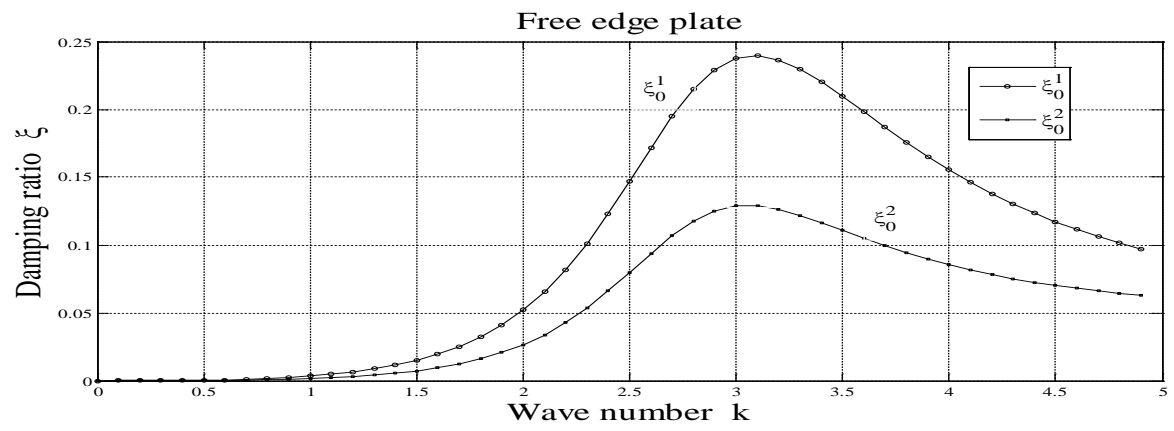

Fig.5. Damping ratio $\xi_{m}^{n}$ vs wave number $k$ for a free edge plate

In Figs. $6,7,8$ and 9 the frequency parameter $C_{\omega}$, defined as $C_{\omega}=\omega \cdot a^{2} \sqrt{\frac{\rho_{p} h}{D}}$, is presented as function of the relative thickness $\frac{t}{h}$ of the plate, with $\frac{t}{h}=0$ for zero thickness, for the modes 1 and 2. It can be observed a great reduction in the frequency parameter when the thickness tends to a small value that in the limit would correspond to the case of a diaphragm or membrane. Also in the same figures, the frequency parameter for the plate in vacuum is presented. In this case the parameter is independent of the plate thickness, therefore the figures show the effect of the fluid on this coefficient.

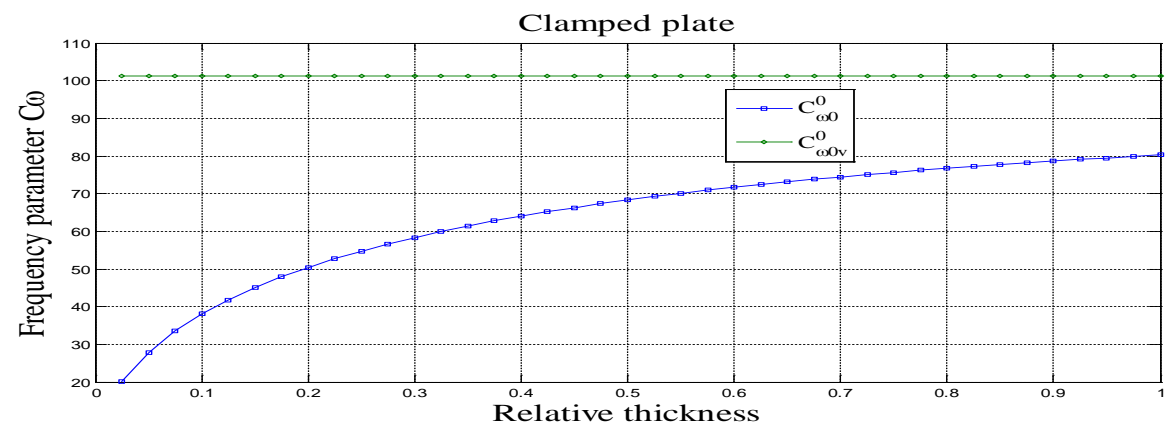

Fig.6. Frequency parameter $C_{\omega m}^{n}$ vs relative thickness for a clamped plate for the mode $m=0, n=0$

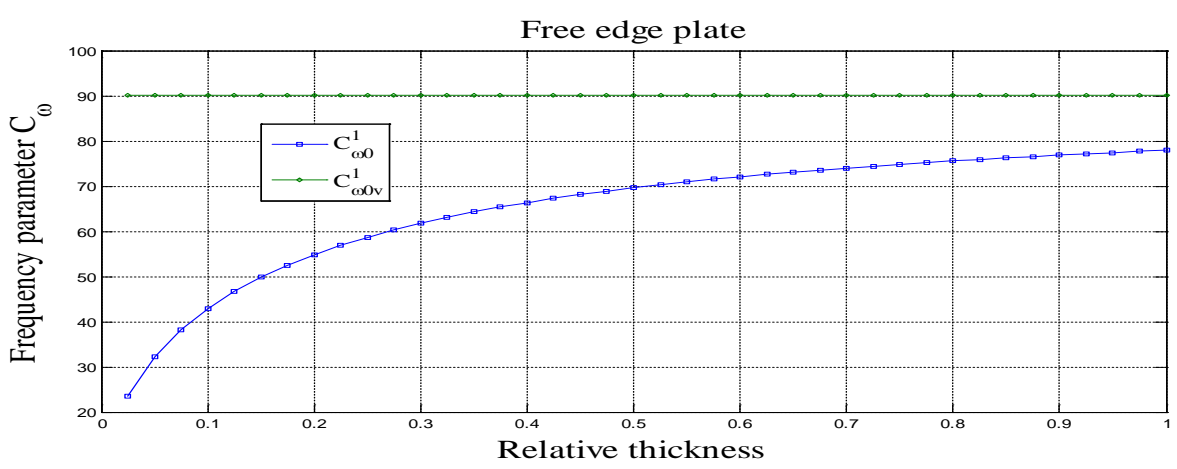

Fig.7. Frequency parameter $C_{\omega m}^{n}$ vs relative thickness for a free edge plate for the mode $m=0, n=1$ 


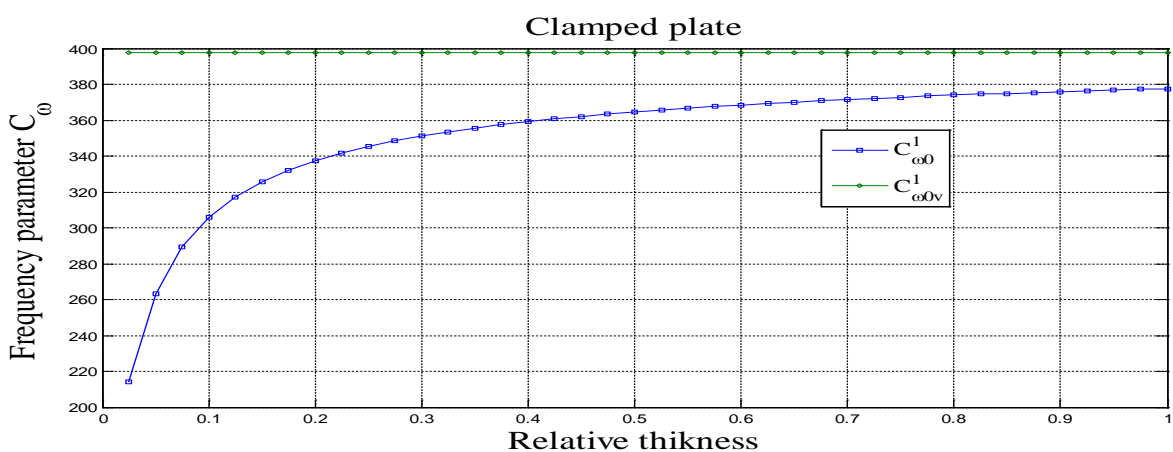

Fig.8. Frequency parameter $C_{\omega m}^{n}$ vs relative thickness for a clamped plate for the mode $m=0, n=1$

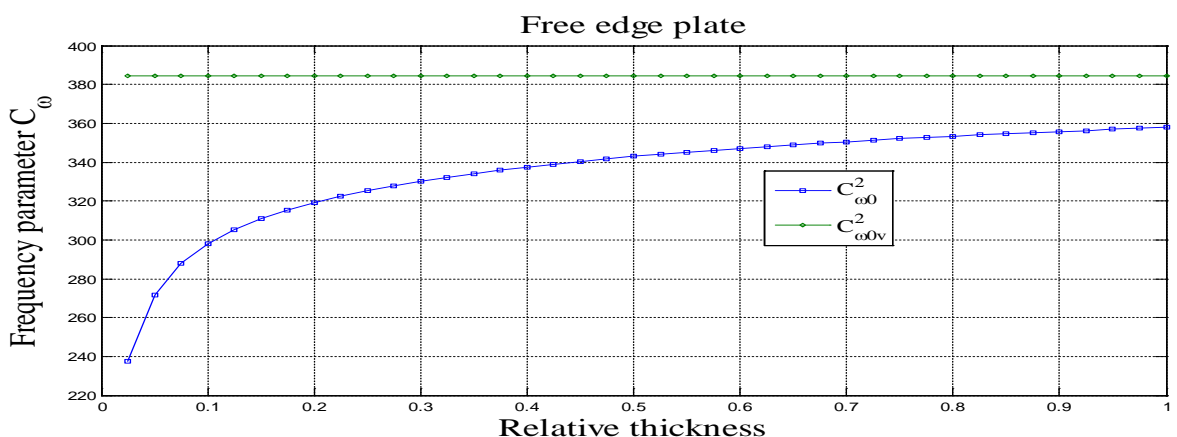

Fig.9. Frequency parameter $C_{\omega m}^{n}$ vs relative thickness for a free edge plate for the mode $m=0, n=2$

In Figs. 10,11,12,13,14 and 15, the damping ratio $\xi$ is presented as a function of the relative density $\frac{\rho}{\rho_{0}}$ of the fluid, with $\rho_{0}=1.225 \frac{\mathrm{kg}}{\mathrm{m}^{3}} \quad$, for different modes corresponding to $n=0,1$ nodal circles and $m=0,1,2$ nodal diameters. In all of cases, $\xi$ increase initially with the density, then presents a maximum for some value of the relative density $\frac{\rho}{\rho_{0}}$ and finally tends to reach a zero value. The range values for this characteristic variation of the damping ratio coefficient with the relative density depends on the mode considered. For a very high value of the density the damping coefficient $\xi$ should go to zero because when the density increases, compressibility effects decrease and so is the phase lag between vibration at the plate and force exerted through the fluid pressure. For each mode there is a density ratio or a speed of sound for which the damping coefficient is maximum. This result could have applications on plates immersed on special media to reduce the level of vibration. 


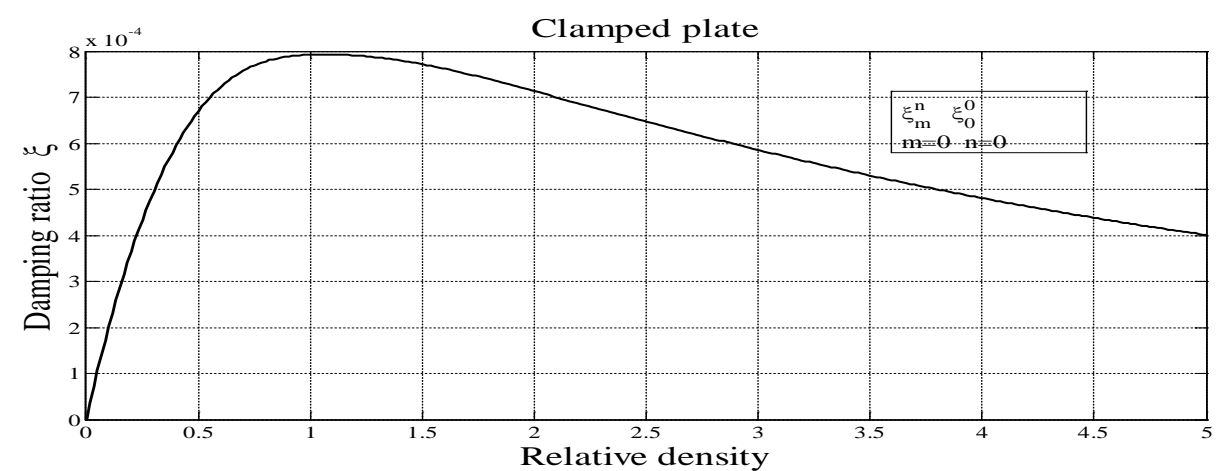

Fig.10. Damping ratio $\xi_{m}^{n}$ vs relative density for a clamped plate for the mode $m=0, n=0$

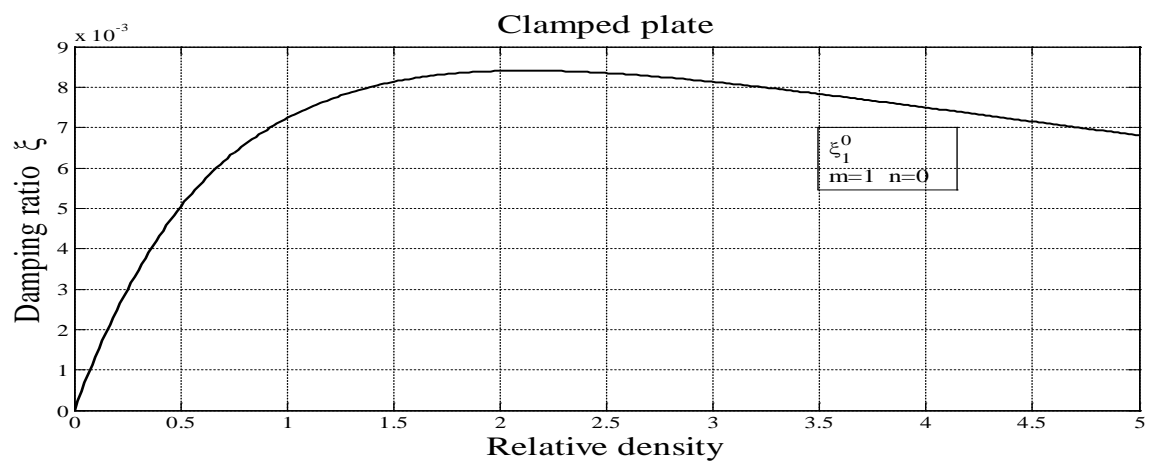

Fig.11. Damping ratio $\xi_{m}^{n}$ vs relative density for a clamped plate for the mode $m=1, n=0$

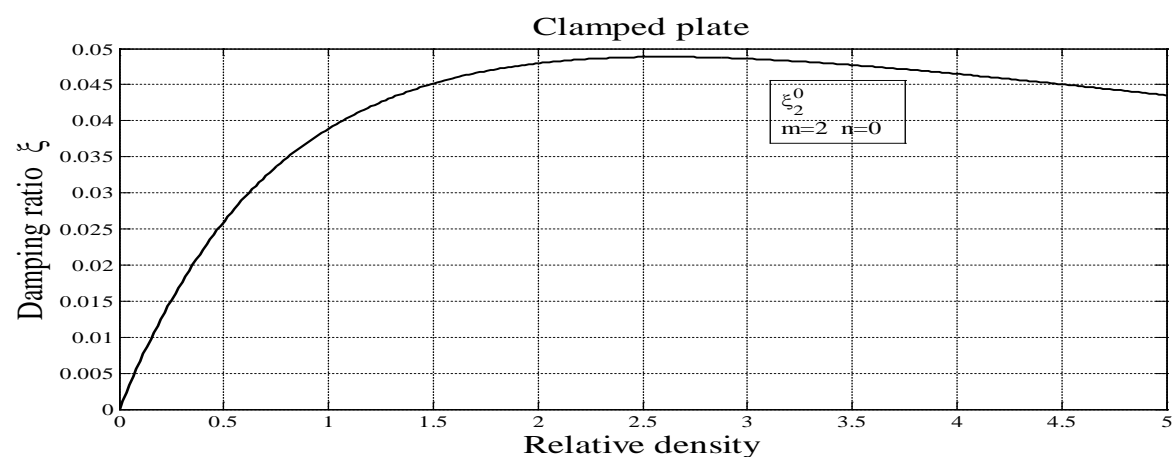

Fig.12. Damping ratio $\xi_{m}^{n}$ vs relative density for a clamped plate for the mode $m=2, n=0$ 


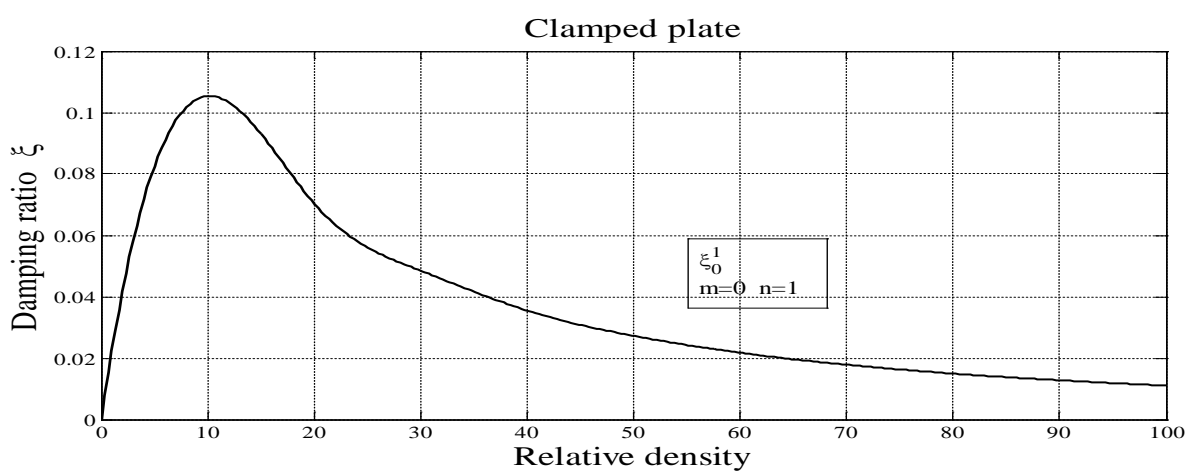

Fig.13. Damping ratio $\xi_{m}^{n}$ vs relative density for a clamped plate for the mode $m=0, n=1$

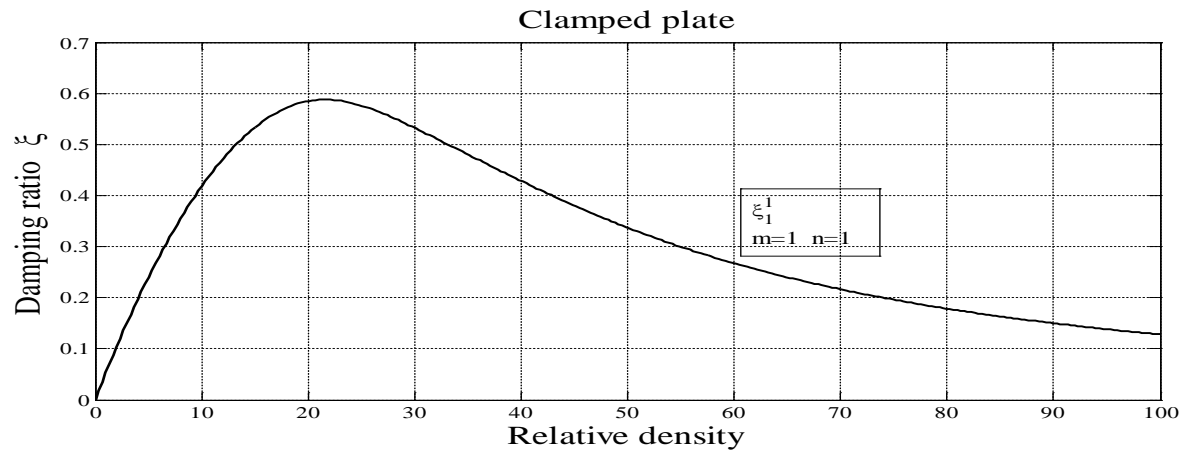

Fig.14. Damping ratio $\xi_{m}^{n}$ vs relative density for a clamped plate for the mode $m=1, n=1$

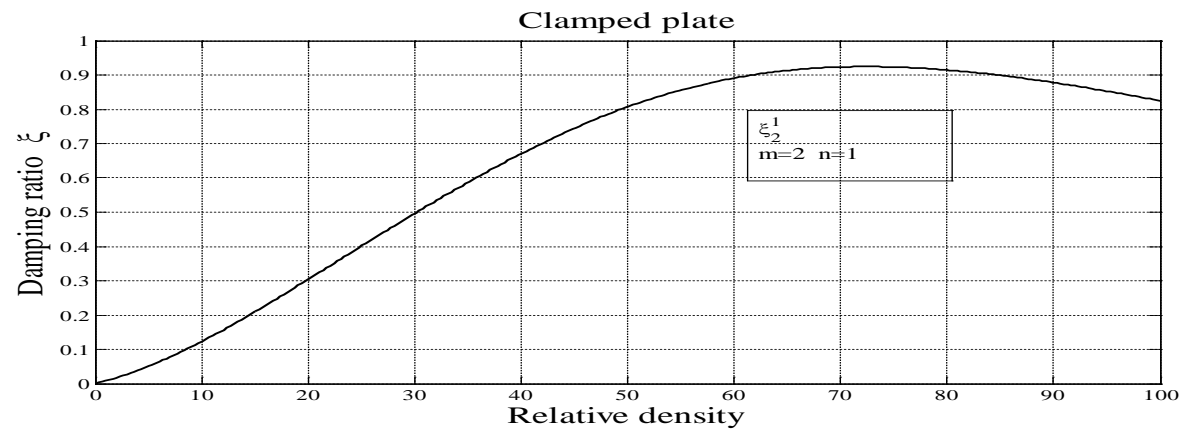

Fig.15. Damping ratio $\xi_{m}^{n}$ vs relative density for a clamped plate for the mode $m=2, n=1$

Next in Table 4, the results of the natural frequencies (in $\mathrm{Hz}$ ) in air (compressible) and in vacuum and the damping ratio are presented for different modes of the same clamped plate. It can be observed that for zero nodal circles, the damping ratio increases when increasing the number of nodal diameters while for one and two nodal circles the damping ratio decreases when increasing the number of nodal diameters 
Table 4. Natural frequencies (in vacuum and air compressible) and Damping ratio for a clamped circular honeycomb plate, radius $a=1 \mathrm{~m}$, thickness $h=1 \mathrm{~cm}$

\begin{tabular}{ccccc}
\hline$m$ & $n$ & $f_{v}$ & $f_{a}$ & $\xi_{m}^{n}\left(\times 10^{-3}\right)$ \\
\hline 0 & 0 & 39.59 & 31.35 & 0.79 \\
1 & 0 & 82.4 & 72.3 & 7.2 \\
2 & 0 & 135.2 & 123 & 38.9 \\
0 & 1 & 154.1 & 144.6 & 50 \\
1 & 1 & 235.7 & 231 & 41.3 \\
2 & 1 & 327.8 & 327.1 & 12.6 \\
0 & 2 & 345.3 & 339.4 & 24.7 \\
1 & 2 & 465.4 & 464.4 & 16.4 \\
2 & 2 & 596.1 & 595.6 & 3.5 \\
\hline
\end{tabular}

Next in Tables 5 and 6 the results of the natural frequencies (in $\mathrm{Hz}$ ) in air (incompressible and compressible) and in vacuum for the same clamped and free edge plate are presented.

Table 5. Natural frequencies for a clamped circular honeycomb plate (in vacuum and air incompressible and compressible), radius $a=1 \mathrm{~m}$, and thickness $h=1 \mathrm{~cm}$

\begin{tabular}{cccccccccccc}
$f_{v 0}^{0}$ & $k=0$ & $f_{a 0}^{0}$ & $k \neq 0$ & $f_{v 0}^{1}$ & $k=0$ & $f_{a 0}^{1}$ & $k \neq 0$ & $f_{v 0}^{2}$ & $k=0$ & $f_{a 0}^{2}$ & $k \neq 0$ \\
\hline 39.6 & 31.59 & 31.37 & 154.1 & 146.82 & 144.57 & 345.3 & 333.16 & 339.4 \\
\hline
\end{tabular}

Table 6. Natural frequencies for a free edge circular honeycomb plate (in vacuum and air incompressible and compressible), radius $a=1 \mathrm{~m}$, and thickness $h=1 \mathrm{~cm}$

\begin{tabular}{|c|c|c|c|c|c|c|c|c|c|c|c|}
\hline$f_{v 0}^{1}$ & $k=0$ & $f_{a 0}^{1}$ & $k \neq 0$ & $f_{v 0}^{2}$ & $k=0$ & $f_{a 0}^{2}$ & $k \neq 0$ & $f_{v 0}^{3}$ & $k=0$ & $f_{a 0}^{3}$ & $k \neq 0$ \\
\hline 35.30 & 30.75 & & 30.63 & 150.6 & 140.79 & & 136.52 & 343.7 & 332.46 & & 337.10 \\
\hline
\end{tabular}

It can be observed that compressibility effects modify about a $2 \%$ the frequencies of the plate in relation to the incompressible fluid assumption. For the first order mode, however, the influence of compressibility is negligible.

\section{Conclusions}

A method has been presented for the computation of the natural frequencies and the acoustic damping ratio of a circular plate surrounded by a compressible fluid of arbitrary density. This method is valid for any type of support condition of the plate and use as input modes those calculated analytically for the vibrating plate in vacuum. The method has been validated with existing tests and with other numerical methods for the cases where compressibility of the fluid is negligible in particular for the case that the fluid is water. In the case of air and light structures, the influence of the fluid has a considerable effect in the reduction of frequency and must be considered. The compressibility effects do not affect very much the results of the frequencies obtained in comparison to the incompressible case; moreover it has been presented the dependency of the acoustic damping ratio with the fluid density and with the reduced frequency for different modes of the plate, showing their influence on this ratio.

\section{References}

Amabili, M. \& Kwak, M.K. [1996],”Free vibrations of circular plates coupled with liquids: revising the Lamb problem”, Journal of Fluids and Structures 10, 743-761

Amabili, M., Pascualini, A. \& Dalpiaz, G. [1995], "Natural frequencies and modes of free edge circular plates vibrating in vacuum or in contact with liquid”, Journal of Sound an 
Vibration 188, 685-699.

Amabili, M., Dalpiaz, G. \& Santolini, [1994], "Free vibration of free edge circular plates immersed in Water", Proceedings of the 12 ${ }^{\text {th }}$ International Modal Analysis Conference 2251: 349.

Askari, E., Jeong, K-H. and Amabili, M., [2013], "Hydroelastic vibration of circular plates immersed in a liquid-filled container with free surface", Journal of Sound and Vibration 332, 3064-3085.

Atalla, N., Nicolas, J. \& Gauthier, C. [1966] "Acoustic radiation of an unbaffled vibrating plate with general elastic boundary conditions”, Journal of the Acoustical Society of America 99 No3:1484-1495.

Berry, A., Guyader, J.L., and Nicolas J. [1990], “A general formulation for the sound radiation from rectangular, baffled plates with arbitrary boundary conditions”, Journal of the Acoustical Society of America 88 (6):2792-2802.

Dowell, E.H. and Hall, K.C. [2001], “Modeling of fluid-structure interaction”, Annual Review of Fluid Mechanics 33:445-490.

Finlayson, B.A. [1972], The method of weighted residuals and variational principles, (Academic Press, Inc., New York).

Fowler, J., Lagerquist, N., \& Leve, H. [1987], “Effect of air in modal tests”, Proceedings of the $5^{\text {th }}$ International Modal Analysis Conference, London.

Gascón-Pérez, M. and García-Fogeda, P. [2014], "Influence of a liquid in the natural frequencies of almost circular plates”, Int. J. Applied Mechanics 6, (5).

Geng, Q. and Li, Y., [2012], "Analysis of dynamic and acoustic radiation characters for a flat plate under thermal environments”, Int. J. Applied Mechanics 04.

He, T. [2015], “On a partitioned strong coupling algorithm for modeling fluid-structure interaction”, International Journal of Applied Mechanics 7 (2) 1550021.

He,T, Zhou, D., Han, Z., Tu, J. and Ma, J. [2014], "Partitioned subiterative coupling schemes for aeroelasticity using combined interface boundary condition method”, International Journal of Computational Fluid Dynamics 28 (6-10) 272-300.

He,T., Zhou, D. and Bao,Y. [2012], "Combined interface boundary condition method for fluid-rigid body interaction”, Computer Methods in Applied Mechanics and Engineering 223: 81-102.

Hou, G., Wang, J. and Layton, A. [2012], "Numerical methods for fluid-structure interaction-A review", Communications Computational Physics 12 (2): 337-377.

Iglesia, F., García-Fogeda, P. López Díez, J. et al [1996], “Effects of the surrounding fluid on the dynamic characteristics of rectangular plate", Machine Vibration 5: 52-61

Junger \& Feit [1972] Sound, structures and their interaction. (M.I.T. Press., London).

Kwak, M.K. [1991], "Vibration of circular plates in contact with water”, Journal of Applied Mechanics 58(2): 480-483.

Lamb Horace, F.R.S. [1920], “On the vibrations of an elastic plate in contact with water”, Proceedings of the Royal Society of London 98: 205-216.

Leissa A.W., [1969], Vibration of plates. NASA SP, Vol. 160 (Scientific and Technical Information Division, NASA).

Lomas, N. S. and Hayek, S.I. [1977], "Vibration and acoustic radiation of elastically supported rectangular plates”, Journal of Sound and Vibration 52 №1: 1-25.

Mangler K.W. [1952], Improper integrals in theoretical aerodynamics, (Aeronautical Research Council Current Papers, London).

McLachlan, N.W. [1932], “The accession to inertia of flexible discs vibrating in a fluid”, Proceeding of the Physical Society 44: 546.

Montero de Espinosa and Gallego Juárez, J.A. [1984], “On the resonance frequencies of water loaded circular plates”, Journal of Sound and Vibration 94 (2): 217-222.

Peake, W.H. \& Thurston, E.G. [1954], "The lowest resonant frequency of a water-loaded circular plate”, Journal of the Acoustical Society of America 26: 166-168.

Rodriguez-Tembleque, L., González, J. A. and Cerrato, A.[2015], "Partitioned solution strategies for coupled BEM-FEM acoustic fluid-structure interaction problems”, Computers and Structures 152:45-58.

Seybert, A.F., Wu, T.W. and Li, W.L. [1993], “A coupled FEM/BEM for fluid-structure interaction using Ritz vectors and eigenvector”, Journal of Vibration Acoustics 115(2):152-158.

Skudrzyk, E. [1971], The foundations of acoustics, (Springer-Verlag, New York).

Tariverdilo, S., Shahmardani, M., Mirzapour, J. and Shabani, R., [2013], “Asymmetric free vibration of circular plate in contact with incompressible fluid”, Applied Mathematical Modelling 37, 228-239.

Zheng, H. and Wei, Z. [2013], "Vibroacoustic analysis of stiffened plates with nonuniform boundary conditions", Int. J. Applied Mechanics 05. 
16 Gascón-Pérez and García-Fogeda 